\title{
Study on the Dispersion of Graphene Oxide in Cement Composites
}

\author{
Qiming Zhang ${ }^{1}$, Changle Fang ${ }^{1}$, Yongkun Mo ${ }^{1}$, Fuming Luo ${ }^{1}$, Wujian Long ${ }^{1, *}$ \\ ${ }^{1}$ School of Shenzhen University, Shenzhen 518000, China \\ Corresponding author e-mail: longwj@szu.edu.cn
}

Keywords: Graphene Oxide, Cement Composites, Study

Abstract: The effects of DAs with different DA to GO mass ratio on the GO dispersion and agglomeration were observed in simulated concrete pore solution (SCPS) and cement pastes. The ions and $\mathrm{pH}$ of cement pastes increased with the amount of cement increasing, which caused the separation of P-HRWR from the GOs. Thus, GOs were absorbed on the surface of the cement particle resulting in GOs sedimentation.

\section{Introduction}

Modern concrete technology is shifting towards high-performance applications with a desire to be sustainable [1]. Nano-materials have been shown to improve the microstructure and comprehensive performance of cement composites [2]. However, due to small particle size, large specific surface area and high surface energy of nano-materials, the use of nano-materials in cement matrix causes the high water consumption and agglomeration [3].

Graphene Oxide (GO) is characterized by good interface contact with cement matrix [4]. However, a high amount of GO in cement matrix does not necessarily give a better performance because excessive GO results in a loss of mechanical performance [5]. The dispersion and distribution of GO in cement pastes is essential to manipulate the properties of the resulting nano-composites. In current studies, the uniform dispersion of GO is achieved by physical and chemical methods [6]. The common physical dispersion methods include ultrasonication, ball milling, and the high shear mill method [6]. The chemical dispersion methods usually use surfactants for surface activation [7].

Currently, most studies focus on the dispersion of GO in aqueous solutions [8], and rarely touch upon its dispersion or re-agglomeration after being added into cement pastes. However, a homogeneous dispersion of GOs in aqueous solutions may not be a guarantee of a uniform dispersion in cement paste [9]. Due to the complex electrolyte environment, the dispersion and stability of GO in the pore solution of concrete can be very different from those in aqueous solutions. Based on the abovementioned, this paper adopts three different dispersing agents and three different GO to DA mass ratios to investigate the dispersion, re-agglomeration of GO in cement composites. Understanding the behavior of GOs in the hydrating cement environment. 


\section{Raw Materials and Experimental Program}

\subsection{Graphene oxide (GO)}

The suspension of GO (3g/L) was prepared by dispersing the graphite oxide powder into water with the help of sonication. Based on the previous study [10], the power and time of ultrasonic were selected $400 \mathrm{~W}$ and 2 hours.

The GO sample was characterized using AFM, TEM and the results are illustrated in Fig. 1. Fig. 1(a) shows that GO sheets exhibited irregular shapes with a dimension of about $0.1 \mu \mathrm{m}$ and a thickness of about $1.7 \mathrm{~nm}$. Fig. 1(b) demonstrates that GO was an almost transparent nanosheet with various wrinkles and folds.
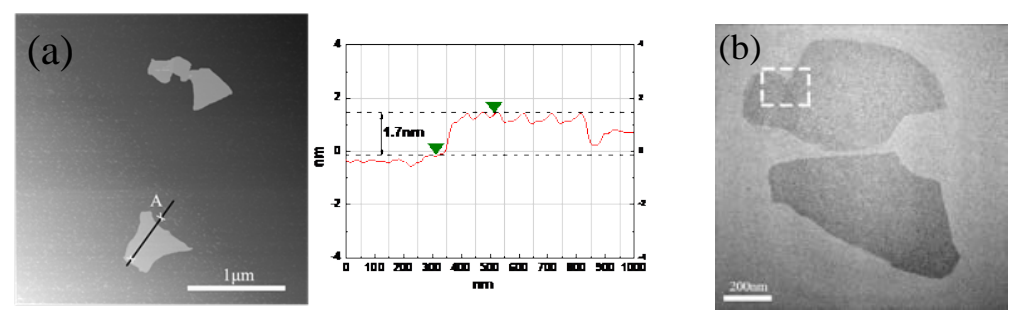

Fig.1The characterization of GO: (a) AFM spectra;(b) TEM image

\subsection{Preparation of GO suspensions}

GO suspensions were prepared to determine the adequate dispersion conditions with regards to DA type and DA to GO mass ratio. GO suspensions were also employed to monitor the stability of the dispersions as a function of $\mathrm{pH}$ in dynamic conditions close to those obtained during cement mixing. To account for the dynamic conditions obtained during cement mixing, cement powder was added to the suspensions in $0.34 \mathrm{~g}$ increments per $100 \mathrm{~mL}$ of suspension to cover a solution $\mathrm{pH}$ range from about 7 ( $\mathrm{pH}$ of the GO aqueous solution with DA) to 11.5 to 12 (pH of cement paste). The stability of the dispersion as a function of $\mathrm{pH}$ was visually monitored.

\section{Results and Discussion}

\subsection{Dispersion state of GO in SCPS}
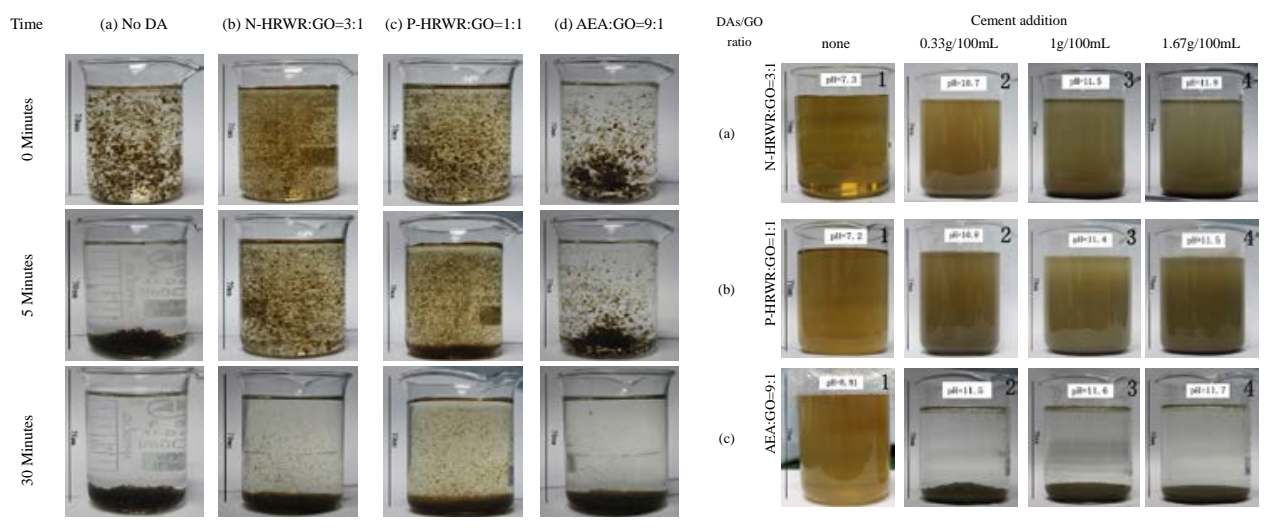

Fig. 2 (1)Dispersion and stability of GO suspensions in SCPS: (2)Effect of cement addition on the stability of GO in aqueous solution at 5.

Figure 2(1) simulates the dispersion state of GO in SCPS, in which the GO to DAs mass ratio 
was based on the best GO dispersion in aqueous solutions. In Fig. 2(1), GO exhibited appreciably greater agglomeration no matter whether DAs were present or not. When there was no DA, almost all the GO separated from SCPS mixed solution after 30 minutes resulting in sedimentation. A few remaining GO suspensions were seen when DAs were present. These results provide the evidence that dispersing agents had a positive effect on GO dispersion in SCPS. During the dispersion process of GO in SCPS solution, cations in SCPS (i.e., $\mathrm{Ca}^{2+}, \mathrm{Na}^{+}, \mathrm{K}^{+}$, etc.) competed with GO for the polymer and combined with GO at the same time, which resulted in sedimentation [11].

\subsection{Effect of cement addition on GO dispersion}

A decrease in dispersion quality of the GO suspensions was observed with increasing cement addition. The separation of the GO agglomerates from solution and then the formation of GO agglomerations were seen when the suspension $\mathrm{pH}$ reached a value of 11 or higher. The process of separation is shown in Figs. 2(2) $a_{3}, a_{4}, b_{3}$, and $b_{4}$ where the top of the solution became light in color compared to Figs. 2(2) $a_{1}, a_{2}, b_{1}$ and $b_{2}$, respectively. In Figs. 2(2) $c_{2}-c_{4}$, the solution turned colorless, and GO uniformly experienced agglomeration and sedimentation. These results agreed with the findings reported by Xing et al [12], where a decrease in dispersion as a function of increasing $\mathrm{pH}$ has been reported for CNTs. According to Figs. 2(2) a-b, N-HRWR and P-HRWR were conducive to the dispersion of GO in cement pastes, even when the $\mathrm{pH}$ was greater than 11 compared to AEA. The cement pastes with N-HRWR and P-HRWR still had a majority of GO which did not experience sedimentation, and the solution with N-HRWR (Fig. 2(2)a $\mathrm{a}_{4}$ ) was lighter in color at the top than that with P-HRWR (Fig. 2(2) $\left.\mathrm{b}_{4}\right)$. Moreover, when the $\mathrm{pH}$ exceeded 11, the GO in the cement pastes added with AEA had experienced fully agglomeration and sedimentation (Figs. 2(2) $\left.\mathrm{C}_{2}-\mathrm{C}_{4}\right)$. The abovementioned results illustrate that P-HRWR was the most conducive to GO dispersion in cement pastes among the three DAs. Furthermore, these results inferred that homogeneous dispersion in the aqueous solution is not a guarantee of effective dispersion in the cement pastes.

\section{Conclusion}

Based on the systematically designed experiments, the effects of the chemical environment relating to stability of GO dispersion in SCPS and cement paste environment were investigated. The following conclusions can be made: Compared with the dispersion of GO in SCPS and cement pastes, homogeneous dispersion of GO in aqueous solution did not guarantee uniform dispersion in cement pastes. With the increase of ions and $\mathrm{pH}$, the P-HRWR separated from the GO. Thus, GO were absorbed on the surface of the cement particle, and GO sedimentation generated.

\section{Acknowledgements}

The authors gratefully acknowledge the financial support provided by the National Natural Science Foundation of China (No.51578341, No. 51278306).

\section{References}

[1] Sanchez F, Sobolev K. Nanotechnology in concrete - A review, Constr. Build Mater. 24 (2010) 2060-2071.

[2] Chang T P, Shih J Y, Yang K M, et al. Material properties of Portland cement paste with nano-montmorillonite, J. Mater. Sci. 42 (2007) 7478-7487.

[3] Shang Y, Zhang D, Yang C, et al. Effect of graphene oxide on the rheological properties of cement pastes, J. Constr. Build. Mater. 96 (2015) 20-28.

[4] Chuah S, Pan Z, et al. Nano reinforced cement and concrete composites and new perspective from graphene oxide, 
J. Constr. Build. Mater. 73 (2014) 113-124.

[5] $\mathrm{Li} \mathrm{W,} \mathrm{Li} \mathrm{X,} \mathrm{et} \mathrm{al.} \mathrm{Effects} \mathrm{of} \mathrm{graphene} \mathrm{oxide} \mathrm{on} \mathrm{early-age} \mathrm{hydration} \mathrm{and} \mathrm{electrical} \mathrm{resistivity} \mathrm{of} \mathrm{Portland} \mathrm{cement}$ paste, Constr. Build. Mater. 136 (2017) 506-514.

[6] Gao Y, Ren X, Tan X, et al. Insights into key factors controlling GO stability in natural surface waters, J. Hazard. Mater. 335 (2017) 56-65.

[7] Lv S, Zhang J, Zhu L, et al. Preparation of cement composites with ordered microstructures via doping with graphene oxide nanosheets and an investigation of their strength and durability, Mater. 9 (2016) 924.

[8] Stephens C, Brown L, Sanchez F. Quantification of the re-agglomeration of carbon nanofiber aqueous dispersion in cement pastes and effect on the early age flexural response, Carbon. 107 (2016) 482-500.

[9] Rana S, Fangueiro R. A Review on nanomaterial dispersion, microstructure, and mechanical properties of carbon nanotube and nanofiber reinforced cementitious composites, J. Nanomater. 2013 (2013) 1-19.

[10] Li H, Zhang M, Ou J. Abrasion resistance of concrete containing nano-particles for pavement, Wear. 260 (2006) 1262-1266.

[11] Ma P, Siddiqui N, et al. Dispersion and functionalization of carbon nanotubes for polymer-based nanocomposites: A review, Compos. Part A. 41 (2010) 1345-1367.

[12] Grunlan J, Liu L, Regev O. Weak polyelectrolyte control of carbon nanotube dispersion in water, J. Colloid Interf. Sci. 317 (2008) 346-349. 